\section{UF FiLORIDiDA}

IFAS Extension

\title{
Turfgrass Science
}

\section{Virginia Buttonweed Biology and Management in Turf ${ }^{1}$}

Darcy E. P. Telenko, J. Bryan Unruh, Barry J. Brecke, and Ramon Leon²

Virginia buttonweed (Diodia virginiana) is a spreading perennial herb with hairy, branched stems. Leaves are opposite, elliptic to lance shaped, sessile, and joined across the stems by a membrane. The membrane has a few "hair-like" projections. Flowers are white and tubular with four lobes at each axil along the stem.
The flower usually only has two sepals. Fruit form at each axil and are green, elliptically shaped, hairy, and ridged. Reproduction occurs via seed, roots, and stem fragments. Virginia buttonweed favors moist to wet sites and is found from New Jersey west to Missouri and south into the Gulf Coast states.
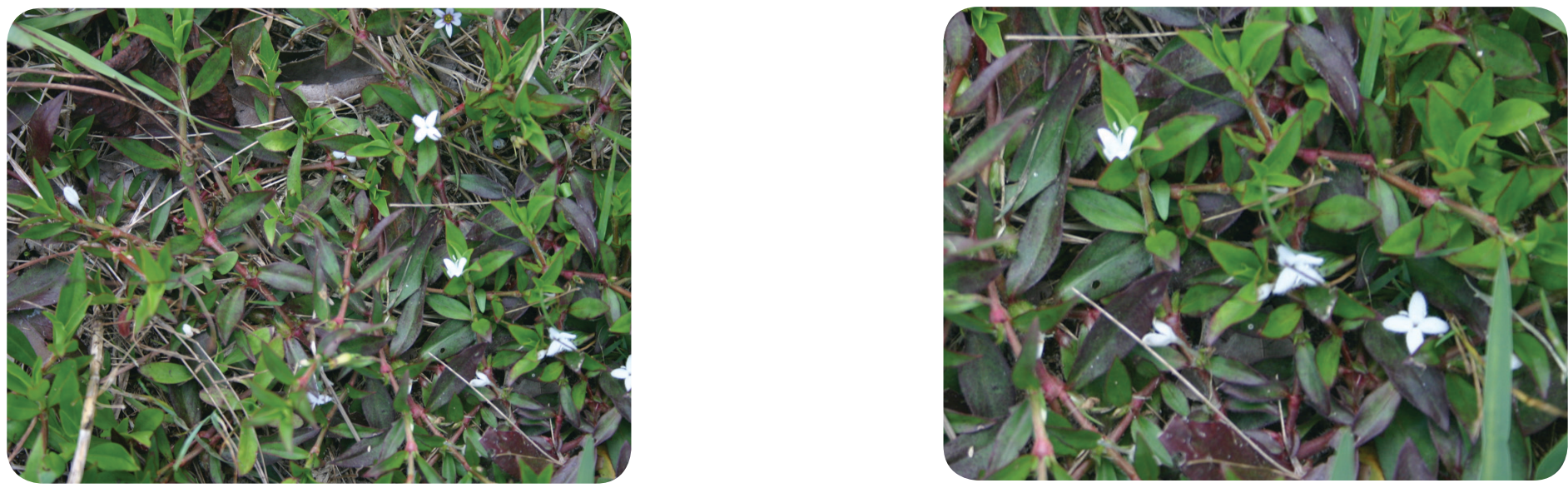

\section{Herbicide options for controlling Virginia buttonweed in Florida turfgrass}

(Always refer to the label for specific uses, application rates and turfgrass tolerance)

BER

Preemergence: none

Postemergence: carfentrazone $+2,4-D$, clopyralid, 2,4-D+dicamba, dicamba, fluroxypyr, thiencarbazone+ iodosulfuron+dicamba, thiencarbazone+floramsulfuron+ halosulfuron, trifloxysulfuron

Preemergence: none

STA Postemergence: ccarfentrazone, 2,4-D+dicamba (follow label recommendations to minimize turf injury), dicamba, thiencarbazone+iodosulfuron+dicamba

CENT

Preemergence: none 2,4-D+dicamba, dicamba, fluroxypyr, thiencarbazone+ iodosulfuron+dicamba, triclopyr+clopyralid

BAHI

Preemergence: none

Postemergence: carfentrazone, clopyralid, 2,4-D+dicamba,

\author{
Preemergence: none \\ Postemergence: carfentrazone \\ Preemergence: none \\ Postemergence: carfentrazone+2,4-D, clopyralid \\ 2,4-D+dicamba, dicamba, fluroxypyr, thiencarbazone+ \\ iodosulfuron+dicamba, triclopyr+clopyralid, \\ trifloxysulfuron \\ Preemergence: none \\ Postemergence: carfentrazone, clopyralid,2, 4-D+dicamba, \\ RYE

\section{ZOYS} \\ dicamba, fluroxypyr, triclopyr+clopyralid \\ PASP
}

Postemergence: carfentrazone+2,4-D, clopyralid, dicamba, fluroxypyr, triclopyr+clopyralid

BER=Bermudagrass; STA=St. Augustinegrass; CENT=Centipedegrass; BAHI=Bahiagrass; PASP=Seashore paspalum; ZOYS=Zoysiagrass; RYE=Perennial rye winter overseed

Refer to the publication Pest Control Guide for Turfgrass Managers at http://turf.ufl.edu/pdf/2012_UF_Pest_Control_Guide.pdf for brand names associated with chemical names listed.

\footnotetext{
${ }^{1}$ This document is ENH1125, one of a series of the Environmental Horticulture Department, Florida Cooperative Extension Service, Institute of Food and Agricultural Sciences, University of Florida. Original publication date July 2009. Revised February 2013. Visit the EDIS website at http://edis.ifas.ufl.edu.

${ }^{2}$ Darcy E. P. Telenko, postdoctoral research associate; J. Bryan Unruh, professor, Environmental Horticulture Department; Barry J. Brecke, professor, and Ramon Leon, assistant professor, Agronomy Department; West Florida Research and Education Center, Cooperative Extension Service, Institute of Food and Agricultural Sciences, University of Florida, Gainesville, 32611.
} 